\title{
Large-scale Patterning of Hydrophobic Silicon Nanostructure Arrays Fabricated by Dual Lithography and Deep Reactive Ion Etching
}

\author{
Zhibo Ma, Chengyu Jiang*, Weizheng Yuan, Yang He
}

(Received 19 October 2012; accepted 7 December; published online 30 January 2013)

\begin{abstract}
We describe a simple but efficient technique to fabricate large-scale arrays of highly ordered silicon nanostructures. By coupling dual lithography using light of $351.1 \mathrm{~nm}$ wavelength with deep reactive ion etching (DRIE), silicon nanostructures of excellent regularity and uniform coverage were achieved. The proposed nanofabrication method not only simplified the nanofabrication process but also produced highaspect-ratio (higher than 15) nanostructures. The scalloping problem was also controlled by regulating DRIE parameters. The process is rapid, cheap, examined to optimize the fabrication process, and has the potential to be scaled up to large areas. The contact angle of a water droplet atop the surface is larger than $150^{\circ}$. Moreover, by coupling black silicon process with DRIE-based microfabrication, three-dimensional nano/nano dual-scale structures which show robust and stable hydrophobicity have been achieved. This process opens new application possibilities in optical, photoelectric, microelectronic, catalytic and biomedical applications.
\end{abstract}

Keywords: Hydrophobic; Nanostructure arrays; DRIE; High-aspect-ratio

Citation: Zhibo Ma, Chengyu Jiang, Weizheng Yuan and Yang He, "Large-scale Patterning of Hydrophobic Silicon Nanostructure Arrays Fabricated by Dual Lithography and Deep Reactive Ion Etching", Nano-Micro Lett. 5(1), 7-12 (2013). http://dx.doi.org/10.3786/nml.v5i1.p7-12

\section{Introduction}

Fabrication of high density array of high-aspect-ratio nanostructures, especially fabrication of nanopillars, has attracted considerable attention as of late. Nanopillars have already been utilized in wide range of applications such as atom diffraction [1], surface plasmon sensor arrays [2], separation of DNA molecules [3], and nanoimprint lithography fabrication.

Usually, nanoscale patterning techniques are involved in the fabrication of nanostructures. Although the standard and conventional electron beam (e-beam) lithography and focused ion beam (FIB) lithography [4] have high resolution and can produce nanoscale structures, both technologies have the disadvantages of low throughput and being expensive. Nanoimprint lithog- raphy (NIL) [5] replicates patterns in a parallel fashion, and have the advantages of high speed, low cost, large pattern area, and high pattern density, but it still needs a master mold manufactured by means of e-beam or X-ray lithography [6]. Interference lithography is an attractive method for fabricating nanostructures, as it offers several advantages including a large exposure area and high spatial-phase coherence. However, the spatial resolution is limited by the light source. Shorter wavelength lasers and immersion techniques have been used to fabricate nanostructures $[7,8]$, but these two methods require complex sources and have limited exposure area.

Because of the drawbacks of aforementioned techniques, a great deal of efforts has been made to develop commercially viable technologies for nanoscale lithog-

Key Laboratory of Micro/Nano Systems for Aerospace, Ministry of Education, Northwestern Polytechnical University, Xi'an, 710072, China

*Corresponding author. E-mail: zbma@nwpu.edu.cn 
raphy. The most commonly used is nanosphere lithography (NSL) [9-13], which has been demonstrated as an efficient way to produce nanoscale patterns over a large area with high throughput and low cost. Nanospheres can be introduced onto substrates by spin-coating [14] or created during annealing a thin metallic $\mathrm{Au}$ ) layer [15], or linked on wafers using self-assembling [16]. However, the shapes and the sizes of the nanoparticles created by these techniques are not as well defined as the features created by using traditional lithography, and the placing of the nanoparticles is also inexact. At the same time, the self-assembly can be very time consuming [17] and long-ranged order is still not achieved [18].

In order to overcome the drawbacks of above techniques, we present a low-cost approach to fabricate high-aspect-ratio silicon nanostructures by dual lithography and high density plasma reactive ion etching (DRIE), achieving high density uniform coverage of space between nanopillars were controlled using dual lithography. This method is fast and cheap, and could be combined with microstructures. Most importantly, it has potential to be scaled up for large areas. Eventually, nano/nano dual-scal hierarchical structures was fabricated by coupling black silicon process with DRIEbased microfabrication, and contact angle measurement and tilting test results show that the fabricated silicon surfaces were superhydrophobic, and the contact angles are all larger than $150^{\circ}$.

\section{Nanostrucures patterned by dual lithog- raphy}

The pattern size of nanopillars and nanotrenches in a photomask was designed as $1 \mu \mathrm{m} \times 1 \mu \mathrm{m}$ and $1 \mu \mathrm{m} \times 1$ $\mathrm{mm}$, which was the minimum size required for successful patternization using photoresist and a contact aligner (MA6/BA6, Karl SUSS company Ltd., Germany).

Figure 1 shows the overall fabrication process of mak-

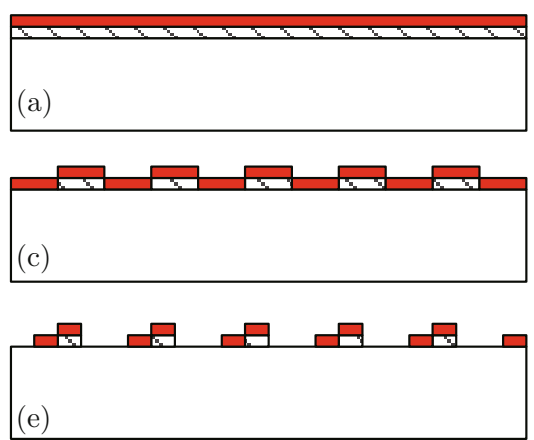
nanostructures over entire 4 inch wafers. The size and

ing silicon nanostructures by dual lithography. The fabrication process started with a 4 inch polished silicon wafer which had been cleaned with a Piranha solution $\left(\mathrm{H}_{2} \mathrm{SO}_{4}: \mathrm{H}_{2} \mathrm{O}_{2}, 4: 1\right.$ by volume) and dehydrated for 10 min at $150^{\circ} \mathrm{C}$. $\mathrm{Cr}(150 \mathrm{~nm})$ was deposited on the silicon surface by a sputtering system. AZ5214E photoresist was then spin-coated at $3000 \mathrm{rpm}$ for $1 \mathrm{~min}$, which resulted in about $800 \mathrm{~nm}$ film thickness. After the spincoating, a soft-bake was done at $110^{\circ} \mathrm{C}$ for $1 \mathrm{~min}$ on a hot plate (Fig. 1(a)).

The substrate was then exposed using microlithography and examined for the estimated time necessary to obtain the appropriate exposure dose, and developed by MF700 developer for $24 \mathrm{~s}$. After the development, the substrate was rinsed with deionized water and blown dry with $\mathrm{N}_{2}$ gas followed by a 1 min hard-bake at $120^{\circ} \mathrm{C}$ on a hot plate. The patterned photoresist was scanned by microscope to assess successful development. Finally, the Cr was etched using wet etchant and the first photoresist served as the etching mask, as shown in Fig. 1(b). These nanostructures with linewidth $1 \mu \mathrm{m}$ also served as a fiducial pattern for subsequent lithography steps.

The first photoresist was then removed with oxygen plasma ashing, followed by the second photoresist spincoated with the same parameters (Fig. 1(c)). Then with the same photomask, the sample was aligned and exposed at a relative offset to the fiducial pattern. The offset may be several hundred nanometers, bigger than $351.1 \mathrm{~nm}$ which is the wavelength of UV light, so the width of unprotected by photoresist is less than $649 \mathrm{~nm}$, as shown in Fig. 1(d).

After the second photoresist developed and a hardbake on a hot plate, the unprotected $\mathrm{Cr}$ was etched using the second photoresist as the etching mask. The photoresist was removed with oxygen plasma ashing, as shown in Fig. 1(e) and Fig. 1(f). Finally, the silicon substrate was then etched by DRIE using the patterned $\mathrm{Cr}$ as an etch mask. After the DRIE, the remaining $\mathrm{Cr}$ was removed and the sample was cleaned with the $\mathrm{Pi}$ ranha solution.

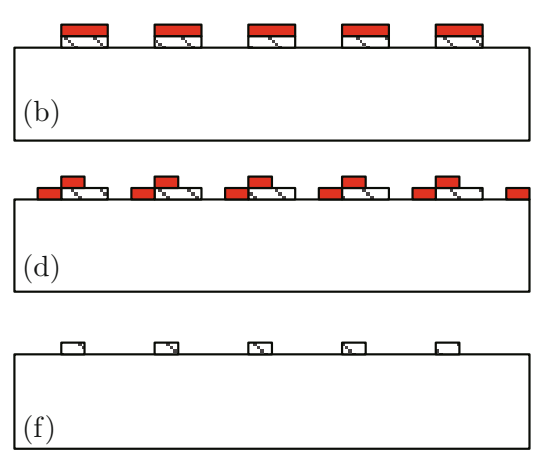

Fig. 1 Dual lithography process of nanostructures patterned. (a) Sputter $150 \mathrm{~nm}$ Cr and photoresist coating. (b) Fist lithography and Cr etching. (c) Second photoresist coating. (d) Second lithography. (e) Cr etching. (f) Photoresist removal and DRIE. 


\section{Results and discussion}

Figure 2 shows SEM images of the fabricated nanopillar or nanotrench arrays of varying heights. The fabricated nanopillars are measured to be about $0.67 \mu \mathrm{m}$ and $0.4 \mu \mathrm{m}$ in width, and $1.96 \mu \mathrm{m}$ and $4.7 \mu \mathrm{m}$ in height, respectively. The aspect ratio of the nanopillars is more than 3 and 11, as shown in Fig. 2(a) and (b). Figure $2(\mathrm{c})$ and (d) show the fabricated nanotrenches, the width are about $0.38 \mu \mathrm{m}$ and $0.66 \mu \mathrm{m}$, their height are about $19.6 \mu \mathrm{m}$ and $4.3 \mu \mathrm{m}$, respectively. The aspect ratio for the nanotrenches is 50 and 6 . However, nanometre scale scallops were formed on the sidewalls of the nanostructures due to the etching and passivation alternating in the DRIE process, as shown in Fig. 2(b) and (d), the peak-to-valley height of scallop is over 87 $\mathrm{nm}$. Although the nanostructures had been achieved by the initially chosen process condition, many studies have shown that the profile affects the scattering loss in optical devices, the electrical performances and flow resistance in fluidic devices [19-22], it is undesirable in some typical applications.

\section{Control of profile roughness}

Although nanostructures had been achieved using an inductively coupled plasma (ICP) etcher with $\mathrm{SF}_{6}+\mathrm{O}_{2}$ etch chemistry and $\mathrm{C}_{4} \mathrm{~F}_{8}$ passivation, the scallops affect the performance of nanostructres, as mentioned before. Table 1 shows the parameters of normal DRIE pro- cess which had been used to fabricate nanostructures in Fig. 2.

Table 1 Study of the DRIE process parameters for nanostructures.

\begin{tabular}{lclc}
\hline \multicolumn{1}{c}{ Etch } & \multicolumn{2}{c}{ Passivate } \\
\hline $\mathrm{SF}_{6}$ flow rate $(\mathrm{sccm})$ & 130 & $\mathrm{C}_{4} \mathrm{~F}_{8}$ flow rate $(\mathrm{sccm})$ & 85 \\
$\mathrm{O}_{2}$ flow rate $(\mathrm{sccm})$ & 13 & & \\
Coil power $(\mathrm{w})$ & 600 & Coil power $(\mathrm{w})$ & 600 \\
Platen power $(\mathrm{w})$ & 12 & Platen power $(\mathrm{w})$ & 0 \\
Cycle time $(\mathrm{s})$ & 11 & Cycle time $(\mathrm{s})$ & 7 \\
Pressure $(\mathrm{m}$ Torr $)$ & & 22 & \\
\hline
\end{tabular}

It has been proven that several parameters, such as $\mathrm{RF}$ power, pressure, and gas mixture, influence the sidewall profile of nanostructures. In order to control the nanoscalloping effect, several parameters had been modified based on plenty of experiments. The etching time was decreased from $11 \mathrm{~s}$ to $8 \mathrm{~s}$, but the passivation time was increased from $7 \mathrm{~s}$ to $10 \mathrm{~s}$, while the other parameters are fixed. Although the etching rate became much slower than before, the roughness of sidewall profiles was improved pronouncedly, as shown in Fig. 3, decreased from tens of nanometers to several nanometers. The result shows that the roughness of sidewall can be diminished by regulating the relative duration of etch time against deposition. Maybe the total number of etch cycles is responsible for the sidewall profile control, as described in reference [23].
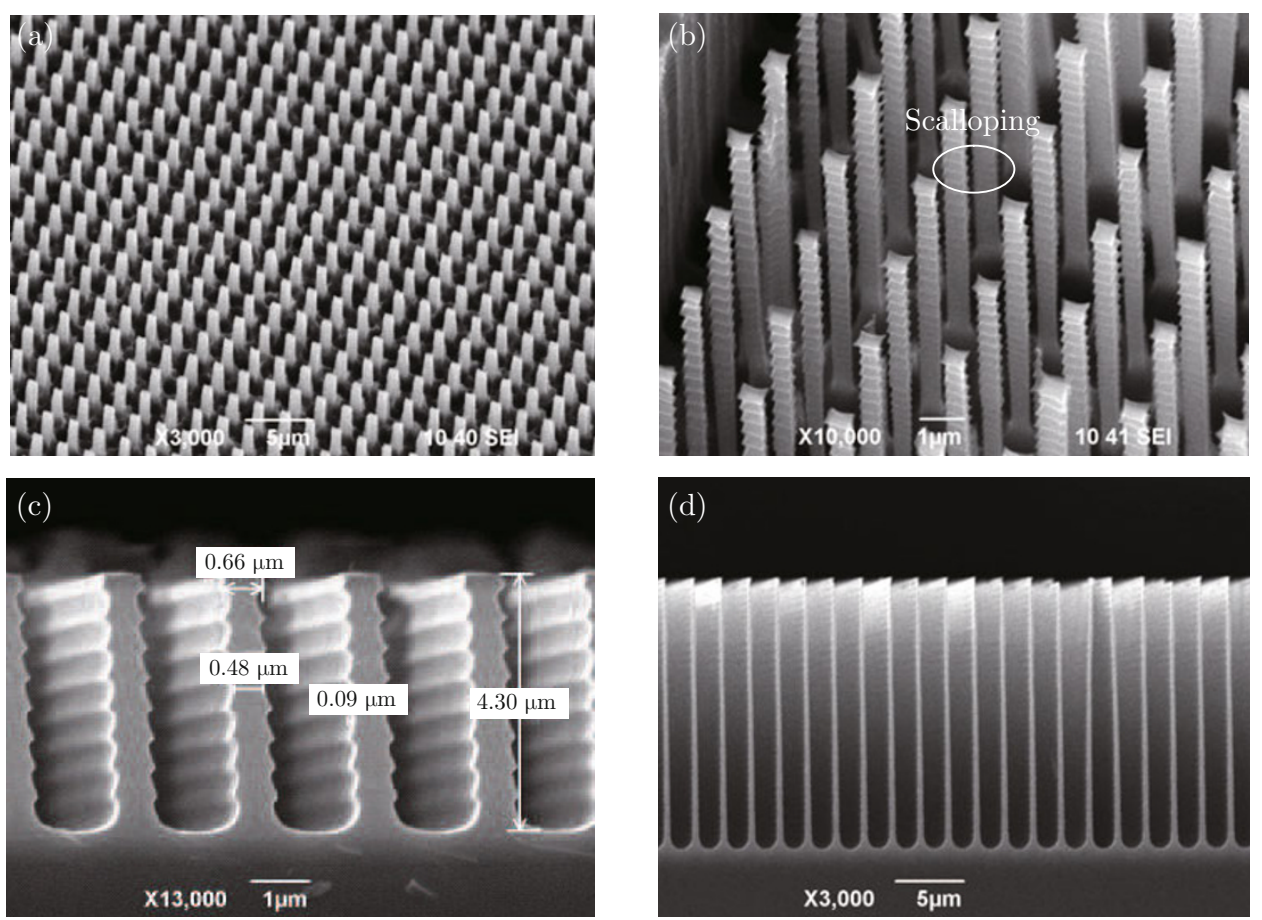

Fig. 2 SEM images of silicon nano-post structures with scalloping effect. (a) Nanopillars with $0.67 \mu \mathrm{m}$ in width, and 1.96 $\mu \mathrm{m}$ in height. (b) Nanopillars with $0.40 \mu \mathrm{m}$ in width and $4.77 \mu \mathrm{m}$ in height. (c) Nanotrenches with $0.66 \mu \mathrm{m}$ in width and $4.30 \mu \mathrm{m}$ in height. (d) Nanotrenches with $0.38 \mu \mathrm{m}$ in width and $19.58 \mu \mathrm{m}$ in height. 


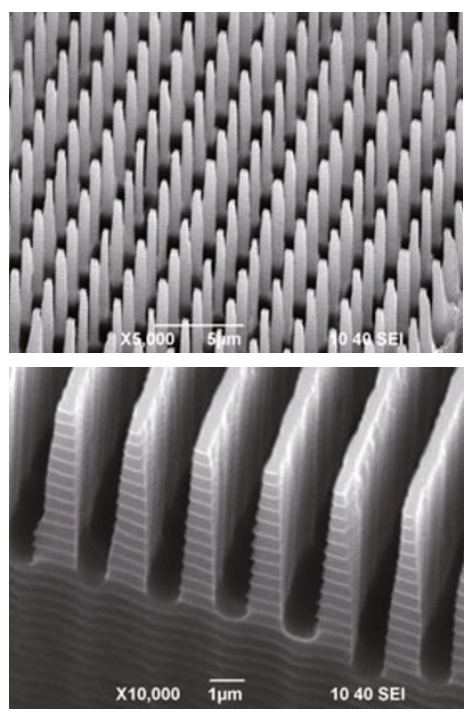

Fig. 3 SEM images of silicon nano-post structures with control of scalloping effect.

\section{Nano/nano dual-scale hierarchical structures fabrication}

Superhydrophobic surface has attracted a considerable amount of attention in recent years. It is suggested that dual-scale roughness patterns at different length is the key factor leading to dramatic superhydrophobicity [24-27]. Highly dense arrays of nanostructures have been successful fabricated by DRIE black silicon [28,29]. However, the uniformity and reproducibility are often more important in some applications. By coupling the dual lithography, DRIE and black silicon, we have developed an effective fabrication process for nano/nano dual-scale hierarchical structures.

Normal DRIE or black silicon is not efficient enough to fabricate dual-scale nanostructures, such as nano/nano dual-scal hierarchical structures. A hybrid etching process between the normal DRIE and black silicon format is required. After the silicon sample was patterned using the aforementioned multilevel lithography, parameters of DRIE including gas flows, coil power, platen power and total etching cycles were adjusted, as listed in Table 2. The perfect hybrid etching regime can be achieved by regulating the gas flow of $\mathrm{SF}_{6}$ and $\mathrm{C}_{4} \mathrm{~F}_{8}$, while keeping other parameters fixed. Figure 4 shows the SEM images of nano/nano dual-scale hierarchical structures fabricated by the hybrid etching process.

Table 2 Study of the DRIE process parameters for nano/nano dual-scal hierarchical structures.

\begin{tabular}{lclc}
\hline \multicolumn{1}{c}{ Etch } & \multicolumn{2}{c}{ Passivate } \\
\hline $\mathrm{SF}_{6}$ flow rate $(\mathrm{sccm})$ & 35 & $\mathrm{C}_{4} \mathrm{~F}_{8}$ flow rate $(\mathrm{sccm})$ & 50 \\
Coil power (w) & 800 & Coil power $(\mathrm{w})$ & 800 \\
Platen power (w) & 8 & Platen power (w) & 8 \\
Cycle time (s) & 4 & Cycle time (s) & 5 \\
Pressure (mTorr) & & \multicolumn{2}{c}{$60(9 \mathrm{~min})$} \\
Total cycles & &
\end{tabular}

\section{Surface characterization results}

Contact angle (CA) and contact angle hysteresis $(\mathrm{CAH})$ of the fabricated nano structure arrays with various geometric parameters were measured, and the results are listed in Table 3. It is clearly seen that all the contact angles are larger than $150^{\circ}$ and quite consistent, and $\mathrm{CAH}$ of them are basically less than $4^{\circ}$. Figure 5 shows CA measurements of the nano structure arrays and nano/nano dual-scal hierarchical structures. It is obvious that the as-prepared silicon surfaces demonstrate high contact angles and low contact angle hysteresis.

Table 3 Contact angle and contact angle hysteresis measurement results

\begin{tabular}{ccc}
\hline sample & $\mathrm{CA}\left(^{\circ}\right)$ & $\mathrm{CAH}\left(^{\circ}\right)$ \\
\hline Nanotrench arrays & 149.7 & 3.6 \\
Nanopillar arrays & 151.4 & 3.2 \\
Nano/nano dual-scal hierarchical arrays & 154.1 & 2.8 \\
\hline
\end{tabular}

To further investigate the hydrophobicity of the fabricated surface, tilting test was implemented and recorded by optical contact angle measurement device. Figure 6 shows two snapped picture of tilting test. It
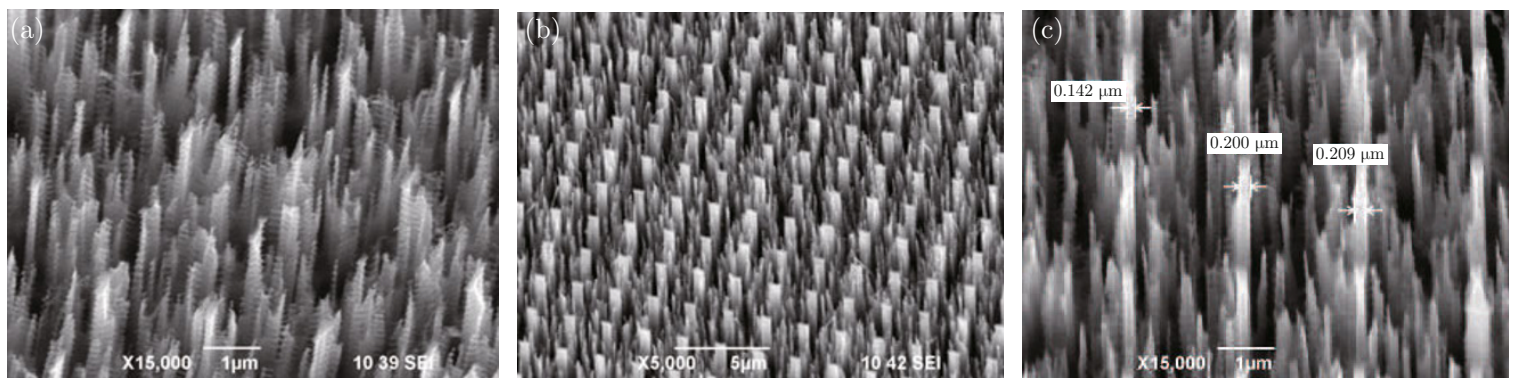

Fig. 4 SEM images of silicon dual-scale structures fabricated by hybrid etching. (a) Black silicon. (b, c) Nano/nano dual-scale structures composed of nanopillar arrays and black silicon, nanopillars are $0.5 \mu \mathrm{m}$ (b) and $0.2 \mu \mathrm{m}$ (c) in width, respectively. 


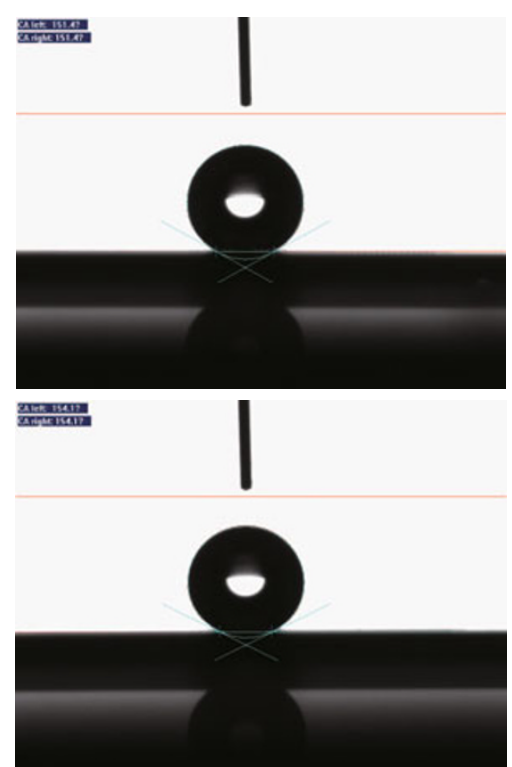

Fig. 5 Static contact angle measurement results. The droplet volume is $12 \mu \mathrm{l}$ and the contact angle is about $151.4^{\circ}$ for nano structures and $154.1^{\circ}$ for nano/nano dual-scal hierarchical structure arrays.

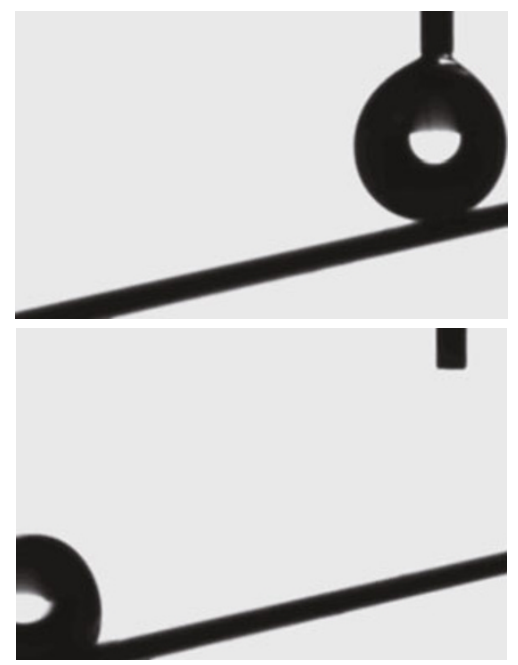

Fig. 6 Snapped picture of the tilting test result.

is seen that water drop easily rolled down when the substrate was titled.

According to above results, it is concluded that the fabricated silicon surfaces with nano pillar arrays were superhydrophobic. The experimental results showed that the average contact angle on flat silicon surfaces is $60^{\circ}$, whilst the water droplet did not bounce back from the flat silicon surfaces. However, the contact angle are larger than $150^{\circ}$ and water droplet bounced back from the surfaces when nano pillar arrays of silicon structures were generated. It is indicated that the hydrophobicity of the fabricated silicon surfaces are enhanced significantly compared with flat silicon surfaces.

\section{Conclusions}

Highly dense arrays of high-aspect-ratio silicon nanopillars have been fabricated using the dual lithography and DRIE. It has also been demonstrated that DRIE together with dual lithography simplifies the fabrication process, and makes it possible to control the size of nanostructures. The DRIE process parameters had also been adjusted to control the roughness of the sidewall profiles of nanostructure. The subsequent simple method of nano/nano dual-scale hierarchical structures' fabrication was also introduced. Contact angle measurement and tilting test results show that the fabricated silicon surfaces were superhydrophobic, and the contact angles are all larger than $150^{\circ}$. This process can also be used to fabricate more complex periodic nanostructures with smooth sidewalls over a large area, it has practical advantages and new application possibilities in optical, photoelectric, microelectronic, catalytic, and biomedical applications.

\section{Acknowledgements}

This work is supported by NPU Foundation for Fundamental Research (NPU-FFR-JCY20130120). The authors would like to acknowledge Dr. Jidong Hou and Jie Kong for useful discussions.

\section{References}

[1] G. Gronniger, B. Barwick and H. Batelaana, "Electron diffraction from free-standing, metal-coated transmission gratings", Appl. Phys. Lett. 87, 124104-124106 (2005). http://dx.doi.org/10.1063/1.2053347

[2] A. D. Ormonde, E. C. Hicks, J. Castillo and R. P. V. Duyne, "Nanosphere lithography: Fabrication of large-area Ag nanoparticle arrays by convective self-assembly and their characterization by scanning UV-visible extinction spectroscopy", Langmuir 201(16), 6927-6931 (2004). http://dx.doi.org/10. 1021/la0494674

[3] Y. C. Chan, Y. K. Lee and Y. Zohar, "Highthroughput design and fabrication of an integrated microsystem with high aspect-ratio sub-micron pillar arrays for free-solution micro capillary electrophoresis", J. Micromech. Microeng. 16(4), 699-707 (2006). http://dx.doi.org/10.1088/0960-1317/16/4/005

[4] J. Taniguchi, K. Koga, Y. Kogo and I. Miyamoto, "Rapid and three-dimensional nanoimprint template fabrication technology using focused ion beam lithography", Microelectron. Eng. 83(4-9), 940-943 (2006). http://dx.doi.org/10.1016/j.mee.2006.01.101

[5] L. J. Guo, "Nanoimprint lithography: Methods and material requirements", Adv. Mater. 19(4), 495-513 (2007). http://dx.doi.org/10.1002/adma. 200600882 
[6] G. Sun, T. Gao, X. Zhao and H. Zhang, "Fabrication of micro/nano dual-scale structures by improved deep reactive ion etching", J. Micromech. Microeng. 20(7), 075028 (2010). http://dx.doi.org/10.1088/ 0960-1317/20/7/075028

[7] T. M. Bloomstein, M. F. Marchant, S. Deneault, D. E. Hardy and M. Rothschild, "22-nm immersion interference lithography", Opt. Express 14(14), 6434-6443 (2006). http://dx.doi.org/10.1364/OE.14.006434

[8] H. H. Solak, C. David, J. Gobrecht, V. Golovkina, F. Cerrina and S. O. Kim, "Sub-50 nm period patterns with EUV interference lithography" Microelectron. Eng. 67-87, 56-62 (2003). http://dx.doi.org/ 10.1016/S0167-9317(03)00059-5

[9] C. L. Haynes and R. P. Van Duyne, "Nanosphere lithography: A versatile nanofabrication tool for studies of size-dependent nanoparticle optics", J. Phys. Chem. B 105(24), 5599-5611 (2001). http://dx.doi. org/10.1021/jp010657m

[10] C. L. Cheung, R. J. Nikolić C. E. Reinhardt and T. F. Wang, "Fabrication of nanopillars by nanosphere lithography", Nanotechnology 17(5), 1339-1343 (2006). http://dx.doi.org/10.1088/0957-4484/17/ $5 / 028$

[11] A. V. Whitney, B. D. Myers and R. P. Van Duyne, "Sub-100 nm triangular nanopores fabricated with the reactive ion etching variant of nanosphere lithography and angle-resolved nanosphere lithography", Nano Lett. 4(8), 1507-1511 (2004). http://dx.doi.org/10. $1021 / \mathrm{nl} 049345 \mathrm{w}$

[12] N. Nagy, E. A. Pap, E. Horv'ath, J. Volk, I. B'arsony, A. De'ak and Z. H'orvolgyi, "Large area self-assembled masking for photonic applications", Appl. Phys. Lett. 89(6), 063104-063106 (2006). http://dx.doi.org/10. $1063 / 1.2335668$

[13] A. Sinitskii, S. Neumeier, J. Nelles, M. Fischer and U. Simon, "Ordered arrays of silicon pillars with controlled height and aspect ratio" Nanotechnology 18(30), 305307 (2007). http://dx.doi.org/10.1088/ 0957-4484/18/30/305307

[14] C. T. Black, K. W. Guarini, K. R. Milkove, S. M. Baker, T. P. Russell and M. T. Tuominen, "Integration of self-assembled diblock copolymers for semiconductor capacitor fabrication", Appl. Phys. Lett. 79(3), 409411 (2001). http://dx.doi.org/10.1063/1.1383805

[15] B. J. Y. Tan, C. H. Sow, T. S. Koh, K. C. Chin, A. T. S. Wee and C. K. Ong, "Fabrication of size-tunable gold nanoparticles array with nanosphere lithography, reactive ion etching, and thermal annealing", J. Phys. Chem. B 109(22), 11100-11109 (2005). http://dx. doi.org/10.1021/jp045172n

[16] S. S. Mark, M. Bergkvist, P. Bhatnagar, C. Welch, A. L. Goodyear, X. Yang, E. R. Angert and C. A. Batt, "Thin film processing using S-layer proteins: Biotemplated assembly of colloidal gold etch masks for fabrication of silicon nanopillar arrays", Colloids Surf. B 57(2), 161-173 (2007). http://dx.doi.org/10.1016/ j.colsurfb. 2007.01.015

[17] V. Gowrishankar, N. Miller, M. D. McGehee, M. J. Misner, D. Y. Ryu, T. P. Russell, E. Drockenmuller and C. J. Hawker, "Fabrication of densely packed, wellordered, high-aspect-ratio silicon nanopillars over large areas using block copolymer lithography", Thin Solid Films 513(1-2), 289-294 (2006). http://dx.doi.org/ $10.1016 / j$.tsf.2006.01.064

[18] L. Sainiemi, H. Keskinen, M. Aromaa, L. Luosujarvi, K. Grigoras, T. Kotiaho, J. M. Makela and S. Franssila, "Rapid fabrication of high aspect ratio silicon nanopillars for chemical analysis", Nanotechnology 18(50), 505303 (2007). http://dx.doi.org/10.1088/ 0957-4484/18/50/505303

[19] K. K. Lee, D. R. Lim, L. C. Kimerling, J. Shin and F. Cerrina, "Fabrication of ultralow-loss $\mathrm{Si} / \mathrm{SiO}_{2}$ waveguides by roughness reduction", Optics Lett. 26(23), 1888-1890 (2001). http://dx.doi.org/10.1364/OL. 26.001888

[20] D. Csontos and H. Q. Xu, "Effects of boundary roughness on the conductance of quantum wires", Appl. Phys. Lett. 77(15), 2364-2366 (2000). http://dx.doi. org/10.1063/1.1311606

[21] H. Cao, Z. Yu, J. Wang, J. O. Tegenfeldt, R. H. Austin, E. Chen, W. Wu and S. Y. Chou, "Fabrication of 10 nm enclosed nanofluidic channels", Appl. Phys. Lett. 81(1), 174-176 (2002) http://dx.doi.org/10.1063/ 1.1489102

[22] Z. Yu and S. Y. Chou, "Triangular profile imprint molds in nanograting fabrication", Nano Lett. 4(2), 341-344 (2004). http://dx.doi.org/10.1021/ n10349471

[23] P. Mukherjee, M. Kang, T. H. Zurbuchen and L. J. Guo, "Fabrication of high aspect ratio Si nanogratings with smooth sidewalls for a deep UV-blocking particle filter", J. Vac. Sci. Technol. B 25(6), 2645-2648 (2007). http://dx.doi.org/10.1116/1.2804612

[24] X. F. Gao and L. Jiang, "Biophysics: Water-repellent legs of water striders", Nature 432 (7013), 36 (2004). http://dx.doi.org/10.1038/432036a

[25] Y. M. Zheng, X. F. Gao and L. Jiang, "Directional adhesion of superhydrophobic butterfly wings", Soft Matter 3(2), 178-182 (2007). http://dx.doi.org/10. $1039 / \mathrm{b} 612667 \mathrm{~g}$

[26] W. J. P. Barnes, "Evolution, stable heterozygosity?", Science 318(5848), 202-203 (2007). http://dx.doi. org/10.1126/science. 1150197

[27] Y. M. Zheng, H. Bai, Z. B. Huang, X. Tian, F. Q. Nie, Y. Zhao, J. Zhai and L. Jiang, "Directional water collection on wetted spider silk", Nature 463 (7281), 640-643 (2010). http://dx.doi.org/10.1038/ nature 08729

[28] C. H. Choi and C. J. Kim, "Large slip of aqueous liquid flow over a nanoengineered superhydrophobic surface", Phys. Rev. Lett. 96(6), 066001-066004 (2006). http:// dx.doi.org/10.1103/PhysRevLett.96.066001

[29] P. Hoyer, M. Theuer, R. Beigang and E. B. Kley, "Terahertz emission from black silicon", Appl. Phys. Lett. 93(9), 091106-091108 (2008). http://dx.doi.org/10. 1063/1. 2978096 